\title{
Métodos de clareamento dental para o sucesso clínico: uma análise comparativa de estudos clínicos através de uma revisão integrativa da literatura
}

Dental bleaching methods for clinical success: a comparative analysis of clinical studies through an integrative literature review

Métodos de blanqueamiento dental para eléxito clínico: un análisis comparativo de estudios clínicos a través de una revisión integradora de la literatura

Thaís Santos de Souza

ORCID: https://orcid.org/0000-0003-4301-9978 Universidade Estadual da Paraíba, Brasil E-mail: thaissouza5086@gmail.com Andreza Mirelly de Queiroz ORCID: https://orcid.org/0000-0002-3876-2399 Universidade Estadual da Paraíba, Brasil E-mail: amqueiroz07@gmail.com Fernanda Kelly Costa Tito ORCID: https://orcid.org/0000-0001-7481-2587 Universidade Estadual da Paraíba, Brasil E-mail: ffernandacosttaa@gmail.com

Maria Renata Alves de Araújo ORCID: https://orcid.org/0000-0001-8845-0767 Universidade Estadual da Paraíba, Brasil E-mail: renataalves914@gmail.com

Matheus Harllen Gonçalves Veríssimo ORCID: https://orcid.org/0000-0003-2845-4832 Universidade Estadual da Paraíba, Brasil E-mail: matheusharllen@gmail.com

Suzie Clara da Silva Marques ORCID: https://orcid.org/0000-0002-7598-6260 Universidade Estadual da Paraíba, Brasil E-mail: suzieclara08@gmail.com

Tallys da Rocha Borges Leal ORCID: https://orcid.org/0000-0002-6718-759X Universidade Estadual da Paraíba, Brasil E-mail: leal.odontotallys@gmail.com

Tauany Maria da Rocha Borges Leal ORCID: https://orcid.org/0000-0003-4521-4144 Universidade Estadual da Paraíba, Brasil E-mail: tauanyleal17@gmail.com

\begin{abstract}
Resumo
Atualmente o clareamento dental é um dos procedimentos mais procurados pelos pacientes na odontologia estética. Tendo em vista a variedade de técnicas disponíveis, é necessário que o cirurgião-dentista esteja apto a escolher o melhor método para atingir o sucesso clínico desse procedimento. Objetivo: Realizar uma revisão integrativa da literatura, com ênfase nos estudos clínicos sobre o clareamento dental na clínica odontológica e responder à pergunta norteadora, além de apresentar os melhores métodos para o sucesso clínico. Metodologia: Trata-se de uma revisão integrativa da literatura de natureza qualitativa realizada a partir da pergunta norteadora: quais os principais aspectos para um sucesso clínico no manejo de clareamentos dentais na clínica odontológica? As bases de dados foram: $U$. $S$. National Library of Medicine (NLM - PubMed) e Science Health and Medical Journals (ScienceDirect), utilizando os seguintes descritores em Inglês encontrados no Descritores em ciências da saúde (Decs): Tooth Bleaching (Clareamento dentário), Tooth Bleaching Agents (Agentes de branqueamento dentário) e Methods (métodos). Os critérios de inclusão foram artigos publicados em inglês, português e espanhol; publicações entre janeiro de 2020 e janeiro de 2021 e que abordavam a temática de maneira satisfatória. Foram excluídos artigos que não atendiam aos critérios de inclusão. Foram encontrados 3.761 artigos na íntegra, após aplicação dos critérios de inclusão e leitura especializada, 14 artigos foram selecionados. Resultados e Discussão: Constatou-se que para alcançar o sucesso clínico do clareamento dental é necessário avaliar fatores intrínsecos ou extrínsecos ao agente clareador: A luz LED
\end{abstract}


violeta (405-410nm) pode melhorar a eficácia do clareamento dental, sem aumentar a sensibilidade; a adição de NaF/ TMP no consultório o clareamento não interferiu na eficácia do clareamento, reduziu a desmineralização, penetração, citotoxicidade, e a alteração celular morfológica foi intensa para todos os géis clareadores; a descoloração do MTA foi melhorada igualmente bem entre a pré-aplicação do DBA e pós-branqueamento; Em relação ao creme dental clareador, o pH mais alto produz uma melhor mudança de tonalidade; $10 \%$, antes do clareamento em consultório, não reduziu o risco e a intensidade da sensibilidade dentária e não prejudicou a mudança de cor; o laser não melhorou os resultados em comparação com o não uso de laser durante o clareamento; o polimento das amostras causou alterações significativas na taxa de despolarização e anisotropia da banda de alongamento simétrica do fosfato, e aumentou a taxa de desmineralização; resina de alto volume apresentou pequena mudança de cor; o ProRoot MTA branco tende a causar descoloração em preto e azul. SavDen MTA, formulado com gluconato de lactato de cálcio, pode ser usado para reduzir a descoloração dos dentes no tratamento endodôntico; os agentes de branqueamento foram eficazes e demonstraram influência moderada na percepção estética e no impacto psicossocial dos pacientes. Considerações Finais: o uso da luz ultravioleta, a busca por materiais que ofereçam menor risco de diminuição da dureza do esmalte e a combinação do tratamento caseiro com o de consultório contribuem para o sucesso clínico do clareamento dental. Palavras-chave: Clareamento dentário; Agentes de branqueamento dentário; Métodos.

\section{Abstract}

Currently, tooth whitening is one of the procedures most sought by patients in cosmetic dentistry. In view of the variety of techniques available, it is necessary for the dentist to be able to choose the best method to achieve the clinical success of this procedure. Objective: To carry out an integrative literature review, with an emphasis on clinical studies on tooth whitening in dental clinics and to answer the guiding question, in addition to presenting the best methods for clinical success. Methodology: This is an integrative review of the literature of a qualitative nature based on the guiding question: what are the main aspects for a clinical success in the management of tooth whitening in the dental clinic? The databases were: US National Library of Medicine (NLM - PubMed) and Science Health and Medical Journals (ScienceDirect), using the following descriptors in English found in Health Sciences Descriptors (Decs): Tooth Bleaching, Tooth Bleaching Agents and Methods. The inclusion criteria were articles published in English, Portuguese and Spanish; publications between January 2020 and January 2021 and that addressed the topic in a satisfactory manner. Articles that did not meet the inclusion criteria were excluded. 3,761 articles were found in full, after application of the inclusion criteria and specialized reading, 14 articles were selected. Results and Discussion: It was found that to achieve the clinical success of tooth whitening, it is necessary to evaluate factors intrinsic or extrinsic to the whitening agent: Violet LED light (405-410nm) can improve the effectiveness of tooth whitening, without increasing sensitivity; the addition of NaF / TMP in the office, whitening did not interfere in the efficacy of whitening, reduced demineralization, penetration, cytotoxicity, and the morphological cellular alteration was intense for all bleaching gels; the discoloration of the MTA was improved equally well between pre-application of the DBA and post-bleaching; In relation to whitening toothpaste, the higher $\mathrm{pH}$ produces a better shade change; $10 \%$, before inoffice whitening, did not reduce the risk and intensity of tooth sensitivity and did not impair the color change; the laser did not improve the results compared to not using a laser during bleaching; the polishing of the samples caused significant changes in the rate of depolarization and anisotropy of the symmetric phosphate elongation band, and increased the rate of demineralization; high volume resin showed a small color change; ProRoot white MTA tends to cause black and blue discoloration. SavDen MTA, formulated with calcium lactate gluconate, can be used to reduce discoloration of teeth in endodontic treatment; bleaching agents were effective and showed a moderate influence on the patients' aesthetic perception and psychosocial impact. Final Considerations: the use of ultraviolet light, the search for materials that offer less risk of decreasing the enamel hardness and the combination of home and office treatments contribute to the clinical success of tooth whitening.

Keywords: Tooth whitening; Tooth whitening agents; Methods.

\section{Resumen}

Actualmente, el blanqueamiento dental es uno de los procedimientos más buscados por los pacientes en odontología cosmética. Dada la variedad de técnicas disponibles, es necesario que el odontólogo pueda elegir el mejor método para lograr el éxito clínico de este procedimiento. Objetivo: Realizar una revisión integradora de la literatura, con énfasis en los estudios clínicos sobre blanqueamiento dental en clínicas dentales y dar respuesta a la pregunta orientadora, además de presentar los mejores métodos para el éxito clínico. Metodología: Se trata de una revisión integradora de la literatura de carácter cualitativo basada en la pregunta orientadora: ¿Cuáles son los principales aspectos para un éxito clínico en el manejo del blanqueamiento dental en la clínica dental? Las bases de datos fueron: Biblioteca Nacional de Medicina de EE. UU. (NLM - PubMed) y Science Health and Medical Journals (ScienceDirect), utilizando los siguientes descriptores en inglés que se encuentran en Health Sciences Descriptors (Decs): Tooth Bleaching (Tooth Bleaching), Tooth Bleaching Agents y Métodos. Los criterios de inclusión fueron artículos publicados en inglés, portugués y español; publicaciones entre enero de 2020 y enero de 2021 y que abordó el tema de manera satisfactoria. Se excluyeron los artículos que no cumplieron con los criterios de inclusión. Se encontraron un total de 3.761 artículos, luego de aplicar los criterios de inclusión y lectura especializada, se seleccionaron 14 artículos. Resultados y Discusión: Se encontró que para lograr el éxito clínico del blanqueamiento dental, es necesario 
evaluar factores intrínsecos o extrínsecos al agente blanqueador: La luz LED violeta (405-410nm) puede mejorar la efectividad del blanqueamiento dental, sin aumentar la sensibilidad. ; la adición de NaF / TMP en el consultorio, el blanqueamiento no interfirió en la eficacia del blanqueamiento, redujo la desmineralización, la penetración, la citotoxicidad y la alteración celular morfológica fue intensa para todos los geles blanqueadores; la decoloración del MTA mejoró igualmente bien entre la pre-aplicación del DBA y el post-blanqueo; En relación a la pasta de dientes blanqueadora, el pH más alto produce un mejor cambio de tonalidad; $10 \%$, antes del blanqueamiento en el consultorio, no redujo el riesgo y la intensidad de la sensibilidad dental y no afectó el cambio de color; el láser no mejoró los resultados en comparación con no usar un láser durante el blanqueamiento; el pulido de las muestras provocó cambios significativos en la velocidad de despolarización y anisotropía de la banda de estiramiento simétrica del fosfato y aumentó la velocidad de desmineralización; la resina de gran volumen mostró un pequeño cambio de color; ProRoot white MTA tiende a causar decoloración de negro y azul. SavDen MTA, formulado con gluconato de lactato de calcio, se puede utilizar para reducir la decoloración de los dientes en el tratamiento de endodoncia; Los agentes blanqueadores fueron efectivos y mostraron una influencia moderada en la percepción estética y el impacto psicosocial de los pacientes. Consideraciones finales: El uso de luz ultravioleta, la búsqueda de materiales que ofrezcan menor riesgo de disminuir la dureza del esmalte y la combinación de tratamientos en el hogar y en la oficina contribuyen al éxito clínico del blanqueamiento dental.

Palabras clave: Blanqueamiento dental; Agentes blanqueadores de dientes; Métodos.

\section{Introdução}

$\mathrm{Na}$ sociedade atual, a preocupação com a aparência é uma realidade que leva os indivíduos a buscarem por procedimentos que melhorem a estética, sendo o clareamento dental um dos mais recorrentes entre eles. Esse desejo surge a partir do padrão de beleza imposto pela cultura moderna, no qual os dentes devem ser mais brancos, bem contornados e corretamente alinhados. Partindo disso, alguns estudos apontam que fatores como esses podem interferir nas relações interpessoais e em julgamentos mais positivos que influenciam na autoestima das pessoas (Espíndola-Castro et al., 2020; Câmara et al., 2020).

Nesse contexto da odontologia, o clareamento dental cumpre um papel importante, atuando como um grande aliado para a devolução da satisfação do sorriso e autoestima do paciente, consistindo em um procedimento menos invasivo e frequentemente indicado (Monteiro et al., 2020). Sendo assim, é indispensável que o cirurgião-dentista aprimore seus conhecimentos acerca das técnicas e métodos disponíveis, atentando para as indicações, vantagens e desvantagens, visando a prescrição de um protocolo correto e mais adequado ao seu paciente (Câmara et al., 2020).

Os agentes branqueadores possuem um mecanismo de ação baseado na oxidação de grandes moléculas de cromóforo, responsáveis pela descoloração do esmalte e da dentina. Esse processo pode acarretar mudanças morfológicas do esmalte dentário ocasionando erosões, aumento da porosidade e consequentemente diminuição do conteúdo mineral. Entretanto, esses quadros podem ser revertidos a partir de agentes remineralizantes ricos em cálcio e fosfato (Espíndola-Castro et al., 2020).

Os principais agentes clareadores que são utilizados são: peróxido de hidrogênio e peróxido de carbamida. Os peróxidos consistem em compostos de peso molecular baixo, o que permite que ocorra sua penetração nas porosidades dos tecidos dentais. Estes agentes clareadores realizam a oxidação de macromoléculas nas manchas de dentina e, em seguida, sua quebra em estruturas menores. Após isso, ocorre a difusão para a superfície, causando o clareamento do dente (Espíndola-Castro et al., 2020).

Dessa forma, este artigo científico teve como objetivo realizar uma revisão integrativa da literatura, com ênfase nos estudos clínicos sobre o clareamento dental na clínica odontológica e responder à pergunta norteadora, além de apresentar os melhores métodos para o sucesso clínico. 


\section{Metodologia}

Esta revisão integrativa da literatura possui uma metodologia qualitativa, sendo baseada em Rohter (2007), Souza, Silva e Carvalho (2010) e Pereira et al. (2018), e no desenvolvimento da seguinte pergunta de pesquisa: Quais os principais aspectos para um sucesso clínico no manejo de clareamentos dentais na clínica odontológica?

Para isto, foram utilizadas duas bases de dados eletrônicas: U. S. National Library of Medicine (PubMed) e Science, Health and Medical Journals (ScienceDirect) para pesquisar e identificar estudos que respondessem à pergunta norteadora desta revisão integrativa da literatura. A base de dados foi pesquisada para estudos realizados entre janeiro de 2020 a janeiro de 2021 .

Esta revisão integrativa baseou-se em três etapas: Na primeira etapa foi o estabelecimento dos descritores para ambas as bases de dados, sendo uma com a utilização de MeSHterms (PubMed) e com DeCS (ScienceDirect). Em seguida, segunda etapa, fora feito a busca avançada nas bases e análise do quantitativo dos artigos científicos presentes na íntegra. Logo em seguida, na terceira etapa, foram selecionados os artigos que se adequaram aos critérios de elegibilidade estabelecidos pelos pesquisadores. Na quarta e quinta etapa, os pesquisadores formularam uma tabela descritiva sobre os autores, objetivo da pesquisa, protocolo, resultados e conclusão e em seguida, desenvolvimento da discussão dos artigos científicos para uma análise do melhor manejo do clareamento dental, a fim de se chegar ao sucesso clínico e responder à pergunta norteadora estabelecida no início desta metodologia.

Foram utilizados três descritores para a composição da chave de pesquisa, sendo os seguintes (MeSH/DeCS): Tooth Bleaching; Tooth Bleaching Agents; Methods. Em seguida, os pesquisadores selecionaram os trabalhos com análise no título e resumo, com base nos critérios de elegibilidade. Os critérios de elegibilidade foram os seguintes: artigos publicados em inglês, português e espanhol; publicações entre janeiro de 2020 e janeiro de 2021; artigos que se adequem à temática. Os resultados por análise foram representados na Tabela 1:

Tabela 1 - Seleção dos artigos por análise empregada e estabelecimento dos critérios de inclusão.

\begin{tabular}{ccccc}
\hline & Íntegra & Duplicados & $\begin{array}{c}\text { Adequados aos } \\
\text { critérios }\end{array}$ & Artigos selecionados \\
\hline PubMed & 1.442 & 3 & 8 & 6 \\
\hline ScienceDirect & 2.319 & 14 & 69 & 8 \\
\hline
\end{tabular}

Fonte: Autores (2020).

Portanto, a partir dessa estratégia de busca, foram encontrados ao todo 3.761 trabalhos na íntegra; destes, 17 artigos encontravam-se duplicados nas estratégias de busca, totalizando, assim, 77 trabalhos adequados aos critérios de inclusão, sendo 14 selecionados.

Também foi utilizado o sistema de formulário avançado para busca e seleção dos artigos utilizando conector booleano “AND”. Em seguida, artigos que preencheram os critérios de elegibilidade foram identificados e incluídos na revisão. Os trabalhos que preencheram todos os critérios de seleção foram incluídos no estudo, os que não preencheram os critérios e/ou não se mostraram relevantes foram excluídos.

\section{Resultados e Discussão}

Atualmente, há uma maior procura por agentes clareadores entre os pacientes odontológicos, principalmente no grupo jovem (Chen et al., 2020). Os agentes clareadores são materiais odontológicos utilizados para promover o embranquecimento 
ou recuperar a cor original dos dentes, em que são utilizados como por exemplo o peróxido de hidrogênio e o de carbamida (Kothari et al., 2020).

Dessa forma, é necessária a análise dos fatores preponderantes (intrínsecos ou extrínsecos ao agente clareador) para o sucesso clínico do clareamento dental. Para isso, estabeleceu-se a construção da Tabela 2 aos estudos clínicos selecionados, com formulação das colunas (Autor/Ano; Objetivo do estudo; Protocolo; Resultados; Conclusão).

Tabela 2 - Relatos de casos detalhados em tabela de resultados.

\begin{tabular}{|c|c|c|c|c|}
\hline Autor/Ano & Objetivo do estudo & Protocolo & Resultados & Conclusão \\
\hline $\begin{array}{l}\text { Brugnera et } \\
\text { al., } 2019 .\end{array}$ & $\begin{array}{l}\text { Avaliar clinicamen-te } \\
\text { o efeito da luz de } \\
\text { diodo emitido por luz } \\
\text { violeta (LED) (405- } \\
410 \mathrm{~nm} \text { ) as-sociada ao } \\
\text { clarea-mento dental } \\
\text { em consultório com } \\
\text { peróxido de carba- } \\
\text { mida a } 35 \% \text {, na } \\
\text { mudança de cor e na } \\
\text { sensibilidade do dente. }\end{array}$ & $\begin{array}{l}50 \text { participantes, em um grupo } \\
\text { de tratamento }(\mathrm{n}=25) \text { : G1 - duas } \\
\text { sessões de clareamento de } \\
\text { 30min cada, com intervalo de } 7 \\
\text { dias (D0 e D7); G2 - duas } \\
\text { sessões de clareamento (mesmo } \\
\text { protocolo do G1) associadas à } \\
\text { luz LED violeta, 30min cada } \\
\text { sessão. }\end{array}$ & $\begin{array}{l}\text { O teste exato de Fisher não } \\
\text { mostrou diferenças estatis- } \\
\text { ticamente significativas de } \\
\text { sensibilidade dentária entre os } \\
\text { grupos. Ambos os grupos } \\
\text { relataram alto nível de } \\
\text { satisfação, mas o teste do qui- } \\
\text { quadrado mostrou pacientes } \\
\text { estatisticamente mais } \\
\text { satisfeitos com o clareamento } \\
\text { dental no G2 do que no G1. }\end{array}$ & $\begin{array}{l}\text { A luz LED violeta } \\
(405-410 \mathrm{~nm}) \text { pode } \\
\text { melhorar a eficácia do } \\
\text { clareamento dental, } \\
\text { sem aumentar a sensi- } \\
\text { bilidade. }\end{array}$ \\
\hline $\begin{array}{l}\text { Akabane et } \\
\text { al., 2020. }\end{array}$ & $\begin{array}{l}\text { Avaliar os efeitos do } \\
\text { gluconato de cálcio } \\
\text { (CaGlu), fluoreto de } \\
\text { sódio (NaF), trime- } \\
\text { tafosfato de sódio } \\
\text { (TMP), e NaF / TMP } \\
\text { adicionado ao } \\
\text { peróxido de hidro- } \\
\text { gênio a 35\% (H } 2 \mathrm{O} 2) \text {, } \\
\text { observando a mudança } \\
\text { de cor, dureza do } \\
\text { esmalte, penetração e } \\
\text { citoto- } \\
\text { xicidadetransame- } \\
\text { lodentinal. }\end{array}$ & $\begin{array}{l}\begin{array}{l}\text { Discos de esmalte / dentina } \\
\text { bovino (n }=288) \text { foram }\end{array} \\
\text { divididos de acordo com o gel } \\
\text { clareador: 35\% H2O2; 35\% } \\
\mathrm{H}_{2} \mathrm{O}_{2}+0,05 \% \mathrm{NaF} ; 35 \% \mathrm{H}_{2} \mathrm{O}_{2}+ \\
0,25 \% \mathrm{TMP} ; 35 \% \mathrm{H}_{2} \mathrm{O}_{2} 0,05 \% \\
\mathrm{NaF}+0,25 \% \mathrm{TMP} ; 35 \% \mathrm{H}_{2} \mathrm{O}_{2}+ \\
0,1 \% \mathrm{NaF}+1 \% \mathrm{TMP} \text { e } 35 \% \\
\mathrm{H}_{2} \mathrm{O}_{2}+2 \% \mathrm{CaGlu} \mathrm{O} \text { gel } \\
\text { clareador foi aplicado três vezes } \\
(40 \text { min/ sessão) em intervalos } \\
\text { de } 7 \text { dias entre cada aplicação. }\end{array}$ & $\begin{array}{l}\text { Todos os géis clareadores } \\
\text { mostraram mudanças de cor } \\
\text { significativas após o } \\
\text { tratamento (p 0,001). Perda } \\
\text { mineral e } \mathrm{H}_{2} \mathrm{O}_{2} \text { a penetração } \\
\text { foi menor para } 35 \% \mathrm{H}_{2} \mathrm{O}_{2} / \\
0,1 \% \mathrm{NaF} / 1 \% \text { TMP; } 35 \%< \\
\mathrm{H}_{2} \mathrm{O}_{2} / \mathrm{Já} \text { o CaGlu } 2 \% \\
\text { apresentou valores mais } \\
\text { elevados, em relação aos } \\
\text { demais grupos (p<0,001). A } \\
\text { viabilidade celular era em } \\
\text { torno de } 9 \% \text {, exceto para o gel } \\
\text { clareador contendo } 35 \% \text { de } \\
\mathrm{H}_{2} \mathrm{O}_{2} / 0,1 \% \text { NaF / 1\% TMP } \\
\text { com 12,8\% (p<0,05). A } \\
\text { formação de nódulos de } \\
\text { mineralização foi maior para } \\
\text { os géis contendo NaF/ TMP ou } \\
\text { CaGlu (p<0,05). A alteração } \\
\text { celular mor-fológica foi } \\
\text { intensa para todos os géis } \\
\text { clareadores. }\end{array}$ & $\begin{array}{l}\text { A adição de } \mathrm{NaF} / \\
\text { TMP no consultório o } \\
\text { clareamento não } \\
\text { interferiu na eficácia } \\
\text { do clareamento, } \\
\text { reduziu a } \\
\text { desmineralização do } \\
\text { esmalte, } \mathrm{H}_{2} \mathrm{O}_{2} \text { penetra- } \\
\text { ção e citotoxicidade. }\end{array}$ \\
\hline $\begin{array}{l}\text { Chen et al., } \\
2020\end{array}$ & $\begin{array}{l}\text { Avaliar os efeitos de } \\
\text { curto prazo de bebidas } \\
\text { causadoras sobre a } \\
\text { manchas de } \\
\text { eficácia do clarea- } \\
\text { mento dental no } \\
\text { consultório. }\end{array}$ & $\begin{array}{l}\text { O participante dividido alea- } \\
\text { toriamente em } 3 \text { grupos com } \\
\text { base nas bebidas utilizadas para } \\
\text { o enxágue durante e após o } \\
\text { procedimento de clareamento: } \\
\text { grupo N (água da torneira, grupo } \\
\text { controle), grupo C (café) e grupo } \\
\mathrm{T} \text { (chá). Enxague com as } \\
\text { respectivas soluções por } 30 \text { s, } 4 \\
\text { vezes ao dia durante } 4 \text { semanas. } \\
\text { Todos os participantes } \\
\text { receberam duas sessões de } \\
\text { tratamento clareador no } \\
\text { consultório com peróxido de } \\
\text { hidrogênio 40\% (Opalescence } \\
\text { BOOST PF 40\%, Ultradent); as } \\
\text { sessões possuíram intervalo de } 1 \\
\text { semana. A coloração dentária foi } \\
\text { avaliada por espectrofotômetro } \\
\text { (Easy-shade, Vita ZahnFabrik) }\end{array}$ & $\begin{array}{l}\text { A diferença de significância } \\
\text { no índice de branco (W), } \\
\text { valores de } \triangle \mathrm{E}, \triangle \mathrm{a} * \mathrm{e} \triangle \mathrm{b} * \\
\text { entre os } 3 \text { grupos foi } \\
\text { observada em qualquer } \\
\text { intervalo de tempo (P para } \\
\text { todos }>0,05) \text {. Em T4, o valor } \\
\text { de } \triangle \mathrm{L} * \text { no grupo } \mathrm{C} \text { foi } \\
\text { significa-tivamente menor do } \\
\text { que nos grupos } \mathrm{T} \text { e } \mathrm{N} \text { (P }= \\
0,022 \text { e } \mathrm{P}=0,001 \text {, respecti- } \\
\text { vamente), embora nenhu-ma } \\
\text { diferença significativa nos } \\
\text { valores de } \triangle \mathrm{L} * \text { tenha sido } \\
\text { observada entre os } 3 \text { grupos } \\
\text { em T1 }(\mathrm{P}=0,402) \text {, T2 }(\mathrm{P}= \\
0,643) \mathrm{T} 3(\mathrm{P}=0,177) \text {. Além } \\
\text { disso, nenhu-ma diferença } \\
\text { significativa foi encontrada } \\
\text { nos valores de TS entre os } 3\end{array}$ & $\begin{array}{l}\text { A exposição ao café } \\
\text { ou chá durante o } \\
\text { período de tratamento } \\
\text { clareador não afetou a } \\
\text { eficácia do tratamento. } \\
\text { No entanto, a } \\
\text { exposição ao café após } \\
\text { o tratamento clareador } \\
\text { afetou a eficácia do } \\
\text { tratamento. } \\
\text { exposição a bebidas } \\
\text { que causam manchas } \\
\text { não afetou o TS } \\
\text { induzido pelo } \\
\text { clareamento. }\end{array}$ \\
\hline
\end{tabular}


antes do procedimento clareador (T0), imediatamente após a primeira sessão de clareamento (T1), imediatamente após a segunda sessão de clareamento (T2), bem como uma semana (T3) e três semanas após (T4) o término do clareamento.
Choiet al., Avaliar a descolo2020. ração do dente por materiais à base de silicato de cálcio recentemente desenvolvidos e examinar a pré-aplicação do agente adesivo dentinário (DBA) para prevenir a descoloração causada pelo trióxidomi-neral agregado (MTA).
As raízes de 50 pré-molares foram divididas aleatoriamente em cinco grupos $(n=10)$. O MTA foi colocado nas cavidades dos dentes pertencentes ao ProRoot MTA (MTA) e Grupos RetroMTA (RMTA). Para dentes pertencentes ao ProRoot + DBA (MTA-B) e RetroMTA + DBA (RMTA-B) grupos, o DBA foi aplicado nas cavidades antes da adição do MTA. Os dentes do grupo de controle foram restaurados com resina composta apenas (ou seja, sem MTA).

Avaliar a eficácia do clareamento e a incidência de hipersensibilidade de contato de três tipos de creme dental clareador.

Kim et al., 2020.

Quarenta e nove participantes foram selecionados acima da tonalidade A2 no incisivo central superior (\#11) e canino (\#13), foram randomizados em três grupos: Grupo TW $(n=15)$, contendo o pH 0,75\% (creme dental branco), Grupo VL ( $\mathrm{n}=$ 15), com o pH a $0,75 \%$ (Vussen 7), e o Grupo VH ( $=17)$, contendo o pH 2,8\% (Vussen 28). Os participantes foram instruídos a escovar os dentes manualmente por 3 minutos, três vezes ao dia, durante 12 grupos em qualquer um dos momentos de avaliação (P para todos $>0,05)$. semanas. Foram acompanhados após 4 e 12 semanas. As medições de tonalidade foram realizadas usando um espectrofotômetro (SP), e os dados foram calculados com CIELab $(\triangle E a b *)$ e fórmula CIEDE2000 $(\Delta E 00)$. Além diso, a inspeção visual (VI) utilizando o guia de cores clássico Vitapan também foi realizado e a correlação entre as duas medidas foi analisada, comparando o CIELab ( $\triangle E a b *)$.

Comparar a mudança de cor do dente e o seguinte de satisfação do parti-cipante: tratamentos de

Kothari et clareamento em casa al., 2020. (HB), no consultório (IOB) e combinados (CB) por 14 dias. IOB foi realizado
Um grupo de 105 participantes recebeu tratamentos $\mathrm{HB}, \mathrm{IOB}$ e CB. A HB foi realizada usando bandejas personalizadas e peróxido de carbamida a $10 \%$ usando peróxido de hidrogênio $37,5 \%$ aplicado em 3 ciclos. A mudança na cor do dente foi avaliada visualmente (VC- $\Delta \mathrm{VC})$ e em aparelho de espectrofotometria digital (ES- $\Delta \mathrm{eVS})$.
Após 12 semanas de RetroMTA causou tratamento com MTA, houve significativamente uma diferença significativa menos descolo-ração entre a descoloração no do que ProRoot MTA. Grupos MTA e RMTA (p Pré-aplicação de DBA $<0,05)$. No entanto, nenhuma reduzida descoloração diferença significativa foi cau-sada pelo ProRoot observada entre o RMTA e o MTA. A descoloração RMTA-B grupos ( $p>0,05)$. do MTA foi melhorada igualmente bem entre a préaplicação do DBA e pós-branquea-mento.

Em 12 semanas de $\mathrm{O}$ creme dental acompanhamento, todos os clareador com o pH grupos apresentaram $\Delta E a b *$ mais alto produz uma acima de 3,46 e $\Delta E 00$ acima melhor mudança de de 2,25 para o dente 11. Para o tonalidade do que dente 13 , o $\Delta E a b *$ e o $\Delta E 00$ outra pasta de dente dos grupos VH e do TW só após 12 semanas. estavam acima esses limiares. A mudança de tonalidade variou de acordo com o creme dental e os pontos de acompanha-mento. SP e VI apresen-taram correlação positiva moderada para $\mathrm{L}^{*}, \mathrm{a}^{*}$ $\mathrm{e} \mathrm{b}^{*}$, respectivamente $(\mathrm{P}$ $<0,05)$. A hipersensibilidade de contato não foi significativamente diferen-te entre os grupos $(\mathrm{P}>0,05)$.
CB resultou em diferença de tonalidade significa-tivamente maior no recall de 15 dias $(\triangle \mathrm{VC}$ e $\Delta \mathrm{eVS}$, todos $\mathrm{p} \leq$ $0,046)$. Às 6 recordatório de meses, o grupo $\mathrm{CB}$ demonstrou maior $\triangle \mathrm{eVS}$ em comparação com IOB ( $\mathrm{p}=$ 0,018), mas a diferença não foi significativa entre os mesmos grupos quando se utiliza o VC $(\mathrm{p}=0,051)$. Níveis de satisfação com retidão dos
$\mathrm{O}$ tratamento $\mathrm{CB}$ resultou em uma melhora pronunciada da cor quando comparado com as duas técnicas utilizadas individualmente. Todos os protocolos de clareamento resultaram em melhora significativa da percepção da saúde 


$\begin{array}{lllr}\text { dentes } \quad \text { foram } & \text { bucal } & \text { dos } \\ \text { significativamente } & \text { participantes } & \text { e } \\ \text { melhorados para CB e IOB em } & \text { satisfação com o } & \text { o } \\ \text { geral. } & \text { sorriso e a brancura } \\ & \text { dos dentes. } & \end{array}$

Avaliaram a degra- Os voluntários foram alocados dação do peróxido de hidrogênio (HP) e carbamida (CP) de géis branqueadores em bandejas prépreenchidas (PT) e personalizadas (TC).

Mailart et

al., 2020.

aleatoriamente para tratamentos $(\mathrm{n}=10)$ : PT-HP/OGO (Opalescence GO-10\%); CT-HP/WTC (Classe Branca-10\%) ou CT$\mathrm{CP} / \mathrm{OPF}$ (Opalescence PF-10\%). das bandejas superior e inferior em cada período (linha de base, $15,30,50$ e 120 minutos). A
Foram coletadas amostras de gel concentração de HP foi determinada com o método de titulação de permangês de potássio. A degradação foi calculada com base na concentração inicial de géis. Medidas repetidas ANOVA e teste de Tukey (5\%) foram aplicados.

Ozdemir \& Avaliar as alteraçõ-es Surmelioglu de cor e minerais , 2020. causadas pelas aplicações de agente clareador em diferentes durações na superfície do esmal-te.

$\mathrm{O}$ incisivo central inferior extraído por razões periodontais foi padronizado para espessura de esmalte e dentina após medidas de tomografia computadorizada de feixe cônico (TCFC) em termos de mineral $(n=24)$ e mudança de cor $(n=36)$. Todos os dentes foram divididos em 3 grupos de acordo com diferentes durações de clareamento para medidas de cor $(\mathrm{n}=12)$ e mudança mineral $(\mathrm{n}=8)$. As amostras para alteração mineral foram examinadas com o dispositivo de raio $X$ de espalhamento de energia (EDX, JSM-6390 LV, Jeol Inc., Japão) antes e 2 semanas após a aplicação.

Pessanha et Avaliar o efeito da al., 2020 aplicação de produ-tos clareadores den-tais no esmalte humano foi avaliado por meio de micros-copia Raman pola-rizada, emissão de raios gama induzida por partículas (PI-GE) e teste de dureza Vickers
Em geral, os géis de branqueamento em ban-dejas inferiores degrada-ram mais do que em bandejas superiores. O gel de peróxido de carbamida apresentou menor degrada-ção do que o peróxido de hidrogênio após 2 horas. A degradação do gel de peróxido de hidrogênio de $10 \%$ usado em bandejas prépreenchidas foi maior do que a de bandejas personalizadas após 30 minutos, mas após 120 minutos, a taxa de degradação foi semelhante para ambos.
Géis branqueantes degradados mais rápido em arcos inferiores do que em $\operatorname{arcos}$ supe-riores. $\mathrm{O}$ processo de degradação foi mais rápido nos primeiros 15 minutos de uso e dependia da composição do gel, tempo de uso e posição da bandeja nas arcadas dentárias.
Devido à sua natureza ácida, os produtos de clareamento dentário estão associados a alterações na mineralização do esmalte. Consequentemente, surgiram no mercado produtos que promovem a incorporação de flúor com o objetivo de diminuir a solubilidade da hidroxiapatita no esmalte $\mathrm{e}$ prevenir a desmineralização. Dessa forma, foram comparados quatro produtos comerciais com diferentes concentrações de princípios ativos: peróxido de carbamida $16 \%$ (Opalescence PF® e VivaStyle ${ }^{\circledR}$ ) ou peróxido de hidrogênio 6\% (Opalescence Go PF® e VivaStylePaintOn $\left.{ }^{\circledR}\right)$ e presença ou não de flúor. A informação sobre o estado
No estudo, foi observada diferença estatisticamente significativa entre todos os grupos em termos de mudança de cor em diferentes momentos de medição (p $<0,05)$. Os maiores valores de E00 foram observados no Grupo $3($ E003 = 8,37 $\pm 2,15)$; o menor valor foi observado no Grupo 1 (E001 = 4,74 \pm 1,26).
O clareamento eficaz foi alcançado em todos os grupos de estudo. Quando os resultados são avaliados como versáteis em termos de eficácia de branqueamento e mudança mineral, a duração de bran-queamento mais ideal foi determinada como 40 min (Grupo 2).
Os resultados obtidos As metodologias determinaram uma diminuição estatisticamente significativa da taxa de despolarização, levando a um aumento da mineralização após a aplicação dos produtos clareadores, exceto o VivaStyle ${ }^{\circ}$. Para este grupo, nenhuma variação significativa foi obtida antes e depois do tratamento, provavelmente devido à acidez do produto $(\mathrm{pH}=5,8$ ) Além disso, foi determinado um aumento da concentração de $\mathrm{F}$ nos tecidos dentais para os produtos fluorados. Por outro lado, o polimento do esmalte, necessário para a aplicação do teste de Vickers, incluindo polimento de amostra e remoção de camada aprismática devem ser usadas com cautela e podem influenciar a interpretação dos resultados obtidos com agentes de desmineralização. Embora o polimento das amostras não tenha causado alterações significativas na taxa de despolarização e anisotropia da banda de alongamento simétrica do fosfato, esse tratamento tornou 


\begin{tabular}{|c|c|c|}
\hline & & $\begin{array}{l}\text { cristalino do esmalte foi } \\
\text { fornecida pela determinação da } \\
\text { taxa de despolarização da banda } \\
\text { de alongamento simétrica do } \\
\text { fosfato }(\text { a } 959 \mathrm{~cm}-1) \text {. }\end{array}$ \\
\hline $\begin{array}{l}\text { Rezende et } \\
\text { al., } 2020 .\end{array}$ & $\begin{array}{lr}\text { Avaliar se a aplicação } \\
\text { tópica de nitrato de } \\
\text { potássio a } r & 10 \% \\
\text { aplicado antes } & \text { do } \\
\text { clareamento } & \text { em } \\
\text { consultório é } & \text { eficaz } \\
\text { para reduzir o risco e a } \\
\text { intensidade } & \text { da } \\
\text { sensibilidade dentária } \\
\text { (TS), bem romo } \\
\text { avaliar re re sua } \\
\text { aplicação } & \text { pode } \\
\text { comprometer } & \text { a } \\
\text { mudança de cor. }\end{array}$ & $\begin{array}{l}\text { Metade da arcada superior do } \\
\text { paciente recebeu a aplicação de } \\
\text { nitrato de potássio a } 10 \% \text { ou gel } \\
\text { placebo por } 10 \text { minutos em um } \\
\text { ambiente universitário. } \\
\text { clareamento em consultório foi } \\
\text { realizado em três aplicações de } \\
15 \text { minutos em duas sessões } \\
\text { clínicas (intervalo de } 1 \text { semana) } \\
\text { usando peróxido de hidrogênio a } \\
35 \% \text {. A mudança de cor foi } \\
\text { comparada usando o teste t } \\
\text { pareado. O nível de significância } \\
\text { foi estabelecido em } 5 \% \text {. }\end{array}$ \\
\hline
\end{tabular}

aumentou a suscetibilidade à

erosão, resultando em

diminuição da dureza e

aumento da taxa de despolarização do esmalte.

Não foi observada diferença significativa nos riscos absolutos de TS entre os grupos (RR 5 1,11; IC 95\% $0,97$ a 1,$27 ; \mathrm{P} 5,12)$, que ultrapassou $87 \%$ para ambos os grupos. Da mesma forma, nenhuma diferença nas intensidades de TS foi detectada (P. 05). as amostras mais

sujeitas

desmineralização.

A aplicação tópica de nitrato de potássio a $10 \%$, antes do clareamento em consultório, não reduziu o risco e a intensidade da ST e não prejudicou a mudança de cor.
Avaliar a eficácia do branqueamento da aplicação do laser com tratamento químico em comparação apenas com $\mathrm{o}$ tratamento químico.

Romero et al., 2020.
Realizou-se o ensaio controlado randomizado (RCT), cego único (avaliador), em 24 pacientes randomizados em dois grupos: intervenção a laser e química (12) ou intervenção química sozinho sem laser (12). Os produtos comerciais utilizados foram o gel de peróxido de hidrogênio Whiteness HP® 35\% e o Whitening Laser® II dos equipamentos DCM. A cor foi medida com um espectotômetro antes, imediatamente e 3 dias após o procedimento de branqueamento. Para estabelecer diferenças antes versus após os tratamentos e entre os grupos, foram aplicados os testes $\mathrm{T}$ e qui ${ }^{2}$. Os dados foram analisados estatisticamente com o teste $\mathrm{T}$ do Aluno $(\mathrm{P} \leq 0,05)$.

Avaliar o efeito dos

Tavares et 3M ESPE®), um al., 2020. protocolos de branqueamento na rugosidade superficial (Ra), mudança de cor e micromorfologia superficial de um composto de baixa viscosidade a granel (Filtek Bulk FillFlow,

Quarenta amostras de cada composto (discos de $5 \mathrm{~mm}$ de diâmetro e $2 \mathrm{~mm}$ de espessura) foram divididas aleatoriamente em quatro grupos $(n=10)$, de acordo com o protocolo de branqueamento: a) gel de peróxido de carbamida de $10 \%$ (Opalescência, Produtos Ultradent $\left.{ }^{\circledR}\right)(2 \mathrm{~h} / \mathrm{dia}$, durante 14 dias); b) $40 \%$ de gel de peróxido de hidrogênio composto preenchimento a granel de altaviscosidade (Filtek Bulk Fill, 3M ESPE®) e uma resina composta nanoparticulada convencional (controle) (Filtek Z350 XT, 3M ESPE®). branqueamento, uma vez por semana, $45 \mathrm{~min} / \mathrm{sessão);} \mathrm{c)}$ enxágue de clareamento (ListerineWhitening Extreme ${ }^{\circledR}$, Johnson \& Johnson $\left.{ }^{\circledR}\right) \quad(2$ $\mathrm{min} /$ dia, durante 14 dias); e d) água destilada (controle). As leituras triplicadas $(\mathrm{Ra}$ e cor [parâmetros CIELAB]) antes e amostras foram submetidas a
Ambos os grupos deram resultados clinicamente significativos antes versus após procedimentos de branqueamento $(\mathrm{P} \leq 0,05)$. Ao comparar entre os grupos que aplicam o teste $\mathrm{T}$, não foram encontradas diferenças estatisticamente significativas $(\mathrm{P} \geq 0,05)$ para os três deltas. Ao comparar sensibilidade após branqueamento, não foram encontradas diferenças entre os grupos $(\mathrm{P} \geq 0,05)$.
O uso de laser durante o tratamento de branqueamento não melhorou os resultados em comparação com o não uso de laser.
O $\mathrm{Ra}$ do volume de alta viscosidade significativamente maior do que o dos outros compósitos $(\mathrm{p}<0,05) . \quad \mathrm{Ra}$ aumentou significa-tivamente $(\mathrm{p}<0,05)$ e a superfície tornou-se mais irregular (análise SEM) em todas as resinas compostas, independentemente do protocolo de branqueamento $(\mathrm{p}<0,05)$. O grupo de resina composta de alta viscosidade tinha $\Delta \mathrm{E}(\mathrm{p}<0,05)$ mais baixo do que o grupo de resina composta nanoparticulada imerso em água destilada
Os autores concluíram que as características de cada resina influenciaram significativamente o $\mathrm{Ra}$ mais do que o protocolo de branqueamento. A resina de alto volume apresentou pequena mudança de cor. 


\begin{tabular}{|c|c|c|c|c|}
\hline & & $\begin{array}{l}\text { depois do contato com } \\
\text { protocolos de branqueamento. A } \\
\text { micromorfologia foi analisada } \\
\text { em um microscópio eletrônico } \\
\text { de varredura (SEM). Os } \\
\text { parâmetros ra e de cor }(\Delta \mathrm{L}, \Delta \mathrm{a}, \\
\Delta \mathrm{b} \text { e } \Delta \mathrm{E}) \text { foram analisados por } \\
\text { modelos lineares generalizados } \\
(\alpha=0,05) .\end{array}$ & & \\
\hline $\begin{array}{l}\text { Yang et al., } \\
2020 .\end{array}$ & $\begin{array}{l}\text { Investigar a des- } \\
\text { coloração de dois } \\
\text { materiais biocerâ- } \\
\text { micos diferentes e os } \\
\text { efeitos } \\
\text { branqueamento do } \\
\text { terno. }\end{array}$ & $\begin{array}{l}\text { Trinta pré-molares } \\
\text { mandibulares com canal único } \\
\text { foram extraídos e divididos } \\
\text { aleatóriamente em três grupos (n } \\
\text { Z 10), ProRoot MTA branco, } \\
\text { SavDen MTA e um grupo } \\
\text { controle. Realizou-se a abertura, } \\
\text { limpeza e modelagem do acesso } \\
\text { endodôntico, em seguida os } \\
\text { dentes foram obturados com os } \\
\text { dois materiais biocerâmicos. A } \\
\text { cor dos dentes foi registrada no } \\
\text { início do estudo, dia } 1 \text { e } 1,2,4, \\
6,8,12,16 \text { e } 24 \text { semanas após o } \\
\text { tratamento. Ao final das } 24 \\
\text { semanas, perborato de sódio foi } \\
\text { utilizado para realizar o } \\
\text { clareamento interno. A cor dos } \\
\text { dentes foi registrada em } 1,2 \text { e } 6 \\
\text { semanas subsequentemente. Os } \\
\text { dentes foram medidos usando } \\
\text { um espectrofotômetro Degu- } \\
\text { Dent, e os dados foram } \\
\text { transformados no sistema } \\
\text { Commission Internationale de } \\
\text { l'Eclairage (CIE). }\end{array}$ & $\begin{array}{l}\text { Os dentes tratados com } \\
\text { ProRoot MTA branco } \\
\text { apresentaram alteração de cor } \\
\text { significativa e diminuição do } \\
\text { valor L). O clareamento } \\
\text { interno levou à diminuição do } \\
\text { valor DE) para todos os três } \\
\text { grupos e ao aumento do valor } \\
\text { L). Não houve diferença na } \\
\text { descoloração dentária entre } \\
\text { SavDen MTA e o grupo } \\
\text { controle após a obturação e } \\
\text { clareamento interno. }\end{array}$ & $\begin{array}{l}\text { Em termos de } \\
\text { percepção visual, o } \\
\text { ProRoot MTA branco } \\
\text { tende a causar } \\
\text { descoloração em preto } \\
\text { e azul. SavDen MTA, } \\
\text { formulado com } \\
\text { gluconato de lactato } \\
\text { de cálcio, pode ser } \\
\text { usado para reduzir a } \\
\text { descoloração dos } \\
\text { dentes no tratamento } \\
\text { endodôntico. }\end{array}$ \\
\hline $\begin{array}{l}\text { Goettems et } \\
\text { al.,2021. }\end{array}$ & $\begin{array}{l}\text { Investigar o impacto } \\
\text { do branqueamento } \\
\text { dentário na qualidade } \\
\text { de vida relacionada à } \\
\text { saúde bucal } \\
\text { (OHRQoL) em } \\
\text { adultos usando um } \\
\text { ensaio clínico } \\
\text { randomizado } \\
\text { comparando } \\
\text { branqueamento em } \\
\text { casa e no consultório. }\end{array}$ & $\begin{array}{l}\text { O tratamento domiciliar foi } \\
\text { realizado utilizando } 10 \% \text { de } \\
\text { peróxido de carbamida ( } 2 \mathrm{~h} / \mathrm{dia} \text {; } \\
3 \text { semanas) e um placebo no } \\
\text { escritório, enquanto } 35 \% \text { de } \\
\text { peróxido de hidrogênio foi } \\
\text { usado para no escritório } \\
\text { (1/semana; } 3 \text { semanas) e um } \\
\text { placebo em casa. A cor foi } \\
\text { avaliada utilizando-se o sistema } \\
\text { CIELAB utilizando um } \\
\text { espectrofotômetro digital. A } \\
\text { mudança de cor objetiva e } \\
\text { subjetiva foi estimada pelo } \\
\text { cálculo das unidades } \\
\text { CIEDE2000 ( } \Delta E 00) \text { e guia de } \\
\text { sombra ( } \mathrm{SGU} \text { ), } \\
\text { respectivamente. O OHRQoL } \\
\text { foi medido utilizando-se a } \\
\text { versão brasileira do Perfil de } \\
\text { Impacto em Saúde Bucal } \\
\text { (OHIP-14). }\end{array}$ & $\begin{array}{l}\text { A mudança objetiva de cor foi } \\
\text { observada em casa ( } \mathrm{p}=0,033) \text {, } \\
\text { em comparação com o in- } \\
\text { office ( } \mathrm{p}=0,429) \text {. Os escores } \\
\text { médios do OHIP-14 foram } \\
\text { semelhantes entre os grupos } \\
\text { na linha de base ( } \mathrm{p}=0,368) \text { e } \\
\text { após o tratamento ( } \mathrm{p}=0,695) \text {. } \\
\text { O branqueamento teve um } \\
\text { efeito positivo moderado } \\
\text { sobre o OHRQoL dos adultos, } \\
\text { especialmente no que diz } \\
\text { respeito aos domínios de } \\
\text { desconforto psicológico, } \\
\text { incapacidade social e } \\
\text { pontuação total de OHIP-14. } \\
\text { A percepção de sensibilidade } \\
\text { dentária no grupo que recebeu } \\
\text { tratamento domiciliar foi } \\
\text { negativamente associada ao } \\
\text { OHRQoL ( } \mathrm{p}=0,037) \text {. }\end{array}$ & $\begin{array}{l}\text { Os agentes branquea- } \\
\text { mento foram } \\
\text { altamente eficazes e } \\
\text { demonstraram } \\
\text { influência mode-rada } \\
\text { na percepção estética e } \\
\text { no impacto } \\
\text { psicossocial dos } \\
\text { pacientes. }\end{array}$ \\
\hline
\end{tabular}

Fonte: Autores (2020).

No estudo clínico de Brugnera et al. (2018) utilizaram uma luz ultravioleta (405 - $410 \mathrm{~nm}$ ) associada a um agente clareador de baixa concentração a fim de melhorar o resultado do clareamento no consultório, reduzir a sensibilidade e aumentar a autoestima do paciente. O material de escolha foi o peróxido de carbamida (PC) a 35\% para o branqueamento no consultório. Foi realizado em dois grupos, no grupo 1 apresentou redução média da cor de 0,8 unidades para os incisivos centrais (IC) e 1,0 
unidade para os caninos (C) utilizando o PC por 30 min, em duas sessões (intervalo de 7 dias). $\mathrm{O}$ grupo 2, utilizou o dispositivo de luz LED violeta e resultou numa mudança significativa de cor, IC 2,8 e C 6,1 unidades.

Akabane et al. (2019) relataram que os agentes clareadores podem causar alterações na morfologia do esmalte e diminuir a dureza do esmalte. Todos os géis clareadores levaram à perda de dureza e à maior perda de dureza no esmalte foi aproximadamente entre as profundidades de 0 a $40 \mu \mathrm{m}$ para todos os grupos testados. Porém o gel de branqueamento contendo peróxido de hidrogênio $\left(\mathrm{H}_{2} \mathrm{O}_{2}\right)$, fluoreto de sódio $(\mathrm{NaF})$ e trimetafosfato de sódio (TMP), obteve os valores mais baixos de perda de dureza superficial em comparação ao gel clareador contendo peróxido de hidrogênio, sendo o mais utilizado no dia a dia clínico.

Com relação à pesquisa de Chen et al. (2020), os pesquisadores avaliaram os efeitos de curto prazo de bebidas causadoras de manchas sobre a eficácia do clareamento dental no consultório. Em seus resultados, a exposição no pós-tratamento afeta na eficácia do clareamento dentário. Já Choi et al. (2020) avaliaram a descoloração do dente por materiais à base de silicato de cálcio e a pré-aplicação do agente adesivo dentinário para prevenir esta descoloração, em que o RetroMTA causou menos descoloração do que o ProoRoot, e a aplicação do agente adesivo melhorou no branqueamento do dente.

Kim et al. (2020) avaliaram a eficácia do clareamento e a incidência de hipersensibilidade de contato de creme dental clareador, os quais em seus resultados concluíram que o $\mathrm{pH}$ mais alto produz uma melhor mudança de tonalidade do que outra pasta de dente após as 12 (doze) semanas. No que tange à Kothari et al. (2020), os autores compararam a satisfação do paciente com o clareamento dentário caseiro, de consultório e combinados. Sendo assim, observaram que os tratamentos combinados (caseiro e de consultório) apresentaram uma melhora acentuada no embranquecimento do que ambos individuais.

Ozdemir et al. (2020), em detalhes a interação entre a duração do clareamento e a estrutura do esmalte, com auxílio do dispositivo CBCT para padronizar a espessura do esmalte e da dentina. Para mudança de cor, a medição das amostras foi realizada com aparelho espectrofotômetro (VITA EasyShade 4.0, Alemanha), foi aplicado OpalescenceBoost PF 40\% (UltradentProducts, EUA) por 20 min no Grupo 1, 40 min no Grupo 2 e 60 min no Grupo 3. A fim de determinar a eficácia do clareamento, as medições de cor foram repetidas às 24 horas, 7 e 14 dias após a aplicação. $\mathrm{O}$ clareamento eficaz foi alcançado em todos os grupos de estudo. Mas em termos de eficácia e mudança mineral, a duração de clareamento mais ideal foi determinada como 40 min (Grupo 2).

No estudo de Mailart et al. (2020), experimentaram em seu estudo de crossover a avaliação da degradação do peróxido de hidrogênio (HP) e carbamida (CP) de géis branqueadores em bandejas pré-preenchidas (PT) e personalizadas (TC). O gel de peróxido de carbamida apresentou menor degradação do que o peróxido de hidrogênio após 2 horas. A degradação do gel de peróxido de hidrogênio de $10 \%$ usado em bandejas pré-preenchidas foi maior do que a de bandejas personalizadas após 30 minutos, mas após 120 minutos, a taxa de degradação foi semelhante para ambos.

Na pesquisa de Romero et al. (2020), os autores investigaram a eficiência do branqueamento utilizando o laser associado ao tratamento químico em comparação com o clareamento usando apenas o tratamento químico, nesse estudo, houve a comprovação de que ambos os grupos obtiveram resultados significativos antes e depois do procedimento, portanto concluíram que o uso do laser durante o tratamento de branqueamento não melhorou os resultados.

Já no estudo de Tavares et al. (2020), foi avaliado o efeito dos protocolos de branqueamento na rugosidade superficial (Rs), mudança de cor e micromorfologia superficial, de um composto de baixa viscosidade a granel, um composto de preenchimento a granel de alta viscosidade e uma resina composta nanoparticulada convencional, após a análise observaram que as características de cada resina influenciaram significativamente o Rs mais do que o protocolo de branqueamento e concluíram que a resina de alto volume apresentou pequena mudança de cor.

Em seu estudo, Pessanha et al. (2020) fizeram comparação entre quatro produtos comerciais que possuíam princípios ativos com concentrações diferentes: peróxido de carbamida 16\% (Opalescence PF® e VivaStyle®) ou peróxido de hidrogênio 
6\% (Opalescence Go PF® e VivaStylePaintOn®) e presença ou não de flúor. O estado cristalino do esmalte foi determinado pela da taxa de despolarização da banda de alongamento simétrica do fosfato (a $959 \mathrm{~cm}-1)$. A avaliação do conteúdo e absorção de F foi realizada usando PIGE nos produtos fluorados e no grupo de controle negativo. A microdureza do esmalte foi avaliada pelo teste de Vickers. Os métodos que envolvem polimento e remoção de camada da amostra podem causar alterações nos resultados que são obtidos com agentes desmineralizantes, tendo em vista que esse que as amostras sujeitas a esse tratamento ficaram mais suscetíveis à desmineralização.

Rezende et al. (2020) realizaram a aplicação de nitrato de potássio a 10\% ou gel placebo na metade da arcada superior do paciente. O clareamento de consultório consistiu em 3 aplicações num intervalo de 15 minutos em duas sessões clínicas, que tiveram um intervalo de uma semana, utilizando peróxido de hidrogênio a 35\%. Foi utilizado o teste t pareado para observar a alteração de cor. Desse modo, os autores observaram que esse método do uso do nitrato de potássio a $10 \%$ anterior ao clareamento não diminuiu a suscetibilidade ou intensidade da sensibilidade dentária, e não interferiu na mudança de cor.

No que concerne o estudo de Goettems et al. (2021), investigou-se o impacto do branqueamento dentário na qualidade de vida relacionada à saúde bucal (OHRQoL) dos pacientes submetidos ao branqueamento dentário. Dessa maneira, o tratamento foi dividido em dois grupos, o domiciliar pelo qual foi utilizado $10 \%$ de peróxido de carbamida, e no consultório que utilizou $35 \%$ de peróxido de hidrogênio, ambos, com auxílio de um placebo.

Yang et al. (2020), observou a descoloração de dois materiais biocerâmicos divergentes e os efeitos do branqueamento interno. Para isto, trinta pré-molares mandibulares foram extraídos e divididos em três grupos (ProRoot MTA branco, SavDen MTA e um grupo controle), após o processo preparatório, os dentes foram obturados com os materiais biocerâmicos e ao final dos seis meses o perborato de sódio foi utilizado para realizar o clareamento.

Assim, para Yang et al. (2020), o clareamento interno é um tratamento prático para dentes tratados endodonticamente podendo ajudar a clarear a descoloração, além da confecção da coroa para melhorar a aparência estética. Ademais, observou-se nos resultados de Goettems et al. (2021), que o clareamento influenciou positivamente o (OHRQoL), independentemente do tipo de tratamento (domiciliar ou no consultório), ambos proporcionando melhorias positivas na aparência dentária e funcionais, sendo assim, eficazes na percepção estética e consequentemente nos efeitos psicossociais.

\section{Considerações Finais}

A partir dos estudos incluídos nessa revisão integrativa da literatura percebeu-se que há uma grande abordagem acerca dos métodos para o clareamento dental na clínica odontológica. Partindo disso, observou-se que alguns aspectos podem contribuir para que o procedimento alcance o sucesso clínico, tais como o uso da luz ultravioleta, a busca por materiais que ofereçam menor risco de diminuição da dureza do esmalte e a combinação do tratamento caseiro com o de consultório, entre outros. Assim, um tratamento que atinge o sucesso clínico proporciona não somente um bom resultado estético, mas também incide positivamente no aspecto psicossocial do paciente.

\section{Referências}

Akabane, S. et al. (2020). Evaluation of the aesthetic effect, enamel microhardness and trans-amelodentinal cytotoxicity of a new bleaching agent for professional use containing trimetaphosphate and fluoride. Journal of the Mechanical Behavior of Biomedical Materials, 114: $1751-6161$.

Câmara, J. F. P. et al. (2020). Effect of tooth enamel staining by coffee consumption during at-home tooth bleaching with carbamide peroxide. Revista de Odontologia da UNESP, 49, e20200024. https://doi.org/10.1590/1807-2577.02420

Chen, Y. H. et al. (2020). Short-term effects of stain-causing beverages on tooth bleaching: A randomized controlled clinical trial. Journal of Dentistry. DOI: https://doi.org/10.1016/j.jdent.2020.103318

Choi, Y. L. et al. (2020). Pre-application of dentin bonding agent prevents discoloration caused by mineral trioxide aggregate. BMC Oral Health, 20 : 163. 
Research, Society and Development, v. 10, n. 3, e40910313643, 2020 (CC BY 4.0) | ISSN 2525-3409 | DOI: http://dx.doi.org/10.33448/rsd-v10i3.13643

Espíndola-Castro, L. F. et al. (2020). Evaluation of the effect of tooth polishing on color stability after in-office bleaching treatment. Revista de Odontologia da UNESP, 49, e20200023. https://doi.org/10.1590/1807-2577.02320

Goettems, M. L. et al. (2020). Impact of tooth bleaching on oral health-related quality of life in adults: A triple-blind randomised clinical trial. Journal of Dentistry, 105: 103564 .

Kim, H. J. et al. (2020). Bleaching toothpaste with two different concentrations of hydrogen peroxide: A randomized double-blinded clinical trial. Journal of Dentistry, 103: 103508.

Kothari, S. et al. (2020). A randomized clinical trial investigating three vital tooth bleaching protocols and associated efficacy, effectiveness and participants' satisfaction. Journal of Dentistry, 95(2020): 103322.

Mailart, M.C. et al. (2020). Hydrogen peroxide degradation of bleaching systems with different trays: Randomized clinical trial. American Journal of Dentistry, 33(2): 89-94

Monteiro, D. et al. (2020). Combination of the custom trays bleaching technique with the in-office bleaching and considerations for result maintenance. RGO Revista Gaúcha de Odontologia, 68, e20200003 https://dx.doi.org/10.1590/1981-86372020000032018-0054

Ozdemir, Z. M. \& Surmelioglu, D. (2020). Effects of differentbleachingapplication time ontooth color and mineral alteration. Annals of Anatomy, DOI: https://doi.org/10.1016/j.aanat.2020.151590

Pereira, A. S. et al. (2018). Metodologia da pesquisa científica. UFSM.

Pessanha, S. et al. (2020). Evaluation of the effect of fluorinated tooth bleaching products using polarized Raman microscopy and particle induced gamma-ray emission. SpectrochimicaActa Part A: Molecular and Biomolecular Spectroscopy, 236: 1386-1425.

Rezende, M. et al. (2020). Prior application of 10\% potassium nitrate to reduce postbleaching sensitivity: a randomized triple-blind clinical trial. The Journal of Evidence-Based Dental Practice, 20(2). https://doi.org/10.1016/ j.jebdp.2020.101406

Romero, J. M. M.; Torales, U.A.V. \& Martínez, C.J.V. (2020). Efficacy of laser application in dental bleaching: A randomized clinical controlled trial. American Journal of Dentistry, 33(2): 79-82.

Rother, E. T. (2007). Revisão sistemática X revisão narrativa. Acta paulista de Enfermagem; 20(2):v.

Souza, M. C.; Silva, M. D. \& Carvalho, R. (2010). Revisão integrativa: O que é e como fazer? Einstein, 8(1): 102-6.

Tavares, B. G. et al. (2020). Effect of bleaching protocols on surface roughness and color change of high- and low-viscosity bulk-fill composite resins. Acta Odontológica Latino americana, 33(2): 59-68. 